\title{
Diagnosing transient plasma status: from solar atmosphere to tokamak divertor
}

\author{
A. S. Giunta ${ }^{a^{*}}$, S. Henderson ${ }^{b}$, M. O’Mullane ${ }^{b}$, J. Harrison ${ }^{c}$, J. G. Doyle ${ }^{d}$ and H. P. \\ Summers \\ ${ }^{a}$ RAL Space, Science and Technology Facilities Council, Rutherford Appleton Laboratory, \\ Harwell Oxford, Didcot, OX11 OQX, UK \\ ${ }^{b}$ Department of Physics, University of Strathlyde, \\ 107 Rottenrow, Glasgow G4 ONG, UK \\ ${ }^{c}$ Culham Centre for Fusion Energy, \\ Culham Science Centre, Abingdon, Oxfordshire, OX14 3DB, UK \\ ${ }^{d}$ Armagh Observatory, \\ College Hill, BT61 9DG Armagh, UK \\ E-mail: alessandra.giuntalstfc.ac.uk
}

ABSTRACT: This work strongly exploits the interdisciplinary links between astrophysical (such as the solar upper atmosphere) and laboratory plasmas (such as tokamak devices) by sharing the development of a common modelling for time-dependent ionisation. This is applied to the interpretation of solar flare data observed by the UVSP (Ultraviolet Spectrometer and Polarimeter), on-board the Solar Maximum Mission and the IRIS (Interface Region Imaging Spectrograph), and also to data from B2-SOLPS (Scrape Off Layer Plasma Simulations) for MAST (Mega Ampère Spherical Tokamak) Super-X divertor upgrade. The derived atomic data, calculated in the framework of the ADAS (Atomic Data and Analysis Structure) project, allow equivalent prediction in non-stationary transport regimes and transients of both the solar atmosphere and tokamak divertors, except that the tokamak evolution is about one thousand times faster.

KEYWORDS: Plasma diagnostics - interferometry, spectroscopy and imaging; Spectrometers; Simulation methods and programs

\footnotetext{
* Corresponding author.
} 


\section{Contents}

1. Introduction $\quad 1$

2. Experiments and Theory $r$

2.1 Atomic populations 2

2.2 VUV/ EUV spectroscopic analysis 3

3. Transient modelling $\quad 4$

3.1 Solar atmosphere applications $\quad 5$

3.1.1 Deriving Te and Ne from a transient modelling 5

3.1.2 Diagnosing transient ionisation from observations $\quad 6$

$\begin{array}{ll}3.2 \text { Controlled fusion applications } & 7\end{array}$

$\begin{array}{ll}\text { 3.2.1 Modelling transient ionisation in a tokamak divertor } & 7\end{array}$

4. Discussion and conclusions $\quad 8$

\section{Introduction}

Composite photon emission, for example in emission line spectra, carries the signature of the underlying plasma parameters and so allows inference of the physical and chemical characteristics of the emitting source. While for astrophysical plasmas (e.g. solar atmosphere) observing radiation is virtually the only experimental avenue open to us for the investigation of the source, for laboratory plasmas (e.g. tokamak devices) spectral analysis is supported by other local methods. However for both environments the analysis of plasma from observations of their spectra remains a central tool to gain physical insights.

Although the basic mechanisms of energy input are different in tokamak and solar upper atmosphere plasmas, their spectral emission shows clear similarities of the underlying plasma and physical processes which enable the development of common modelling approaches. Atomic physics provides the link to interpret the spectra in terms of the properties (e.g. temperature, density, atomic processes and dynamic status) of the source from which they are emitted. The close connection between the afore-mentioned astrophysical and laboratory plasmas leads to the possibility of making measurements in a more accessible and controlled Earth-based situation to confirm or support results when applying them to astronomy.

This work focusses on the investigation of a model to analyse and characterise from observations the transient plasma status, concentrating especially on non-equilibrium ionisation. Attention is given to applications which range from the solar upper atmosphere, and in particular solar flares, to tokamak divertor plasmas.

Section 2 discusses the motivation of this work from the experimental and theoretical points of view. The transient modelling is described in Section 3, providing examples of both solar atmosphere and fusion applications. Finally, Section 4 summarises the conclusions. 


\section{Experiments and Theory}

Great advances have been made in studies both of the solar physics and magnetic confinement fusion environments in the past decades. New and forthcoming space-borne solar instruments include the Interface Region Imaging Spectrometer (IRIS) and the Spectral Imaging of the Coronal Environment (SPICE) on-board Solar Orbiter (launch due in 2018) which allow observations up to the relatively dense upper chromosphere/lower transition region with temporal resolution (cadence) oriented to realistic dynamic conditions (e.g. flares).

Simultaneously, interest in the behaviour of a wider range of species in fusion plasma has been growing, mainly due to the new thermonuclear reactor, ITER (International Thermonuclear Experimental Reactor), which is under construction in Cadarache (France), and planning for DEMO (DEMOnstration Power Station), which would follow ITER. For ITER key parts of the inner wall of the device will be composed of tungsten. Of key concern is the shape and behaviour of the low temperature protective divertor plasma where a range of light and medium weight species control conditions. The upcoming MAST (Mega Ampère Spherical Tokamak) Super-X upgrade divertor experiment in the UK is focussed on advanced divertor behaviour. It is the fusion divertor plasma which bears the similarity to the solar upper atmosphere - that is dynamic, finite-density, electron-excited plasma of similar temperatures and significant overlap of species of interest.

The new instrumentation and devices demand a matched precision of atomic modelling and derived spectroscopic techniques incorporating fully the finite density environment and fresh methodology for the detection of non-equilibrium processes as well as confirming equilibrium features.

\subsection{Atomic populations}

The solar upper atmosphere and the tokamak divertor plasmas are optically thin to their own emitted radiation apart from Lyman of hydrogen in tokamaks and a few lines of neutral helium and of some light ions in the solar upper atmosphere case, especially $\mathrm{C}^{1+}, \mathrm{C}^{2+}, \mathrm{O}^{1+}, \mathrm{O}^{2+}, \mathrm{N}^{1+}$. So the local emissivity of a spectrum line is the product of the number density of the upper state of the emitting ion (called the population of the level here) and the A-value for the transition.

The usual simplifying assumption for low density plasma has been that the population of the upper state is increased by electron impact excitation from the ground state of the emitting ion only (since only the ground state population is of sufficient number density) and depleted by spontaneous radiative decay which results in the upper population being many orders of magnitude less than the ground population. This is an oversimplification. It cannot be in the upper chromosphere/lower transition region [11] or in dynamic conditions (e.g. flares), where the electron density can reach value of $10^{12}-10^{13} \mathrm{~cm}^{-3}[16]$ and certainly not in tokamak divertor with densities $\geq 5 \times 10^{13} \mathrm{~cm}^{-3}$. Excitation may occur to more highly excited levels and then cascade down to the upper level of the transition. Also at higher plasma electron densities, a second collision can occur with the upper level, or the more highly excited levels in competition with the radiative transitions. So the upper populations have a second-order dependence on electron density and spectrum line ratios become sensitive to density, selectively, dependent on the available collisional re-distribution and cascade pathways.

Modern population modelling tries to take account of all the collisional and radiative pathways carefully up to sufficiently high levels, called the truncation point, and then makes more approximate estimates for levels above the truncation point. Fortunately, theoretical and experimental results are becoming available and more reliable for many ions and the precision of atomic physics calculations has improved considerably [1]. Nonetheless, misinterpretation of a density sensitivity, a non-Maxwellian plasma or a transient is still common because of poor data or too low a truncation point [10]. This is the finite density problem and it affects also ionisation state through equivalent issues for effective recombination and ionisation processes. The comprehensive multi-process population handling is Collisional-Radiative $(C R)$ theory.

There is a further flawed assumption, namely that the ground states of ions are the starting point for the excitation, redistribution, and cascade progression. As it is well known, the population of the $\mathrm{C}^{2+}(2 \mathrm{~s} 2 \mathrm{p}$ 
$\left.{ }^{3} \mathrm{P}\right)$ term is similar to that of the ground term $\mathrm{C}^{2+}\left(2 \mathrm{~s}^{2}{ }^{1} \mathrm{~S}\right)$ in finite density plasma, so it also must be a starting point for the progression and should be on the same footing as the ground term. Such a level in $C R$ modelling is called a metastable. Metastable populations are large, comparable to ground populations and should be treated with them.

The extension of $C R$ theory to handle this is called Generalised-Collisional-Radiative (GCR) theory. Ground and metastable levels have long relaxation times in a finite density plasma in comparison with true excited levels which are very short-lived. Thus in a spatially or temporarily evolving plasma, excited level populations approach local steady state balance with respect to the instantaneous ground and metastable populations, while the latter are subject to spatial displacements and/or time delays according to the rate of plasma evolution. This is called the metastable problem. In a dynamic plasma such as a solar flare, the ionisation equilibrium assumption [7, 8] cannot be made.

The $G C R$ approach $[2,4,20]$, rather than a simplified coronal (zero density) picture or more complex $C R$ theory, handles comprehensively the issue of density, level truncation and the role of metastable levels, in order to reconstruct and model the observed intensities in a large range of plasma regimes.

This approach has been fully developed in ADAS (Atomic Data and Analysis Structure) [19] and GCR coefficients are available for all elements up to neon and recently extended to silicon [11]. These are exploited in the next sections and subsections.

\subsection{VUV/EUV spectroscopic analysis}

The primary goal in observing the VUV/EUV spectra for the Sun or laboratory plasmas is to learn the physical conditions in the observed region and to investigate why the plasma is in that particular state.

The current progress on fundamental atomic calculations and their inclusion in the GCR modelling allow the improvement of spectroscopic diagnostic techniques for deriving the properties of emitting plasma.

The intensity of radiation, emitted by the Sun or any other astrophysical and laboratory source, depends on the probability that the atoms within the observed plasma are in a condition to make transitions and on the probability that the photons produced by these transitions escape from the volume of the plasma without being reabsorbed. The first aspect is related to the distribution of atomic population among the various energy levels, which is established by collisions with other particles and by radiative processes, and to the atomic probability of the transitions (Sect. 2.1). The second aspect concerns the effect of the interaction of radiation with the plasma and in the optically thin case can be neglected. Therefore, the intensity of a spectral line between two levels $i$ and $j$ is given by the following equation:

$$
I_{j \rightarrow i}=\frac{A(Z)}{4 \pi} \int G_{j \rightarrow i}^{(z)}\left(T, N_{e}\right) \Phi(T) d T
$$

Where $T$ and $N_{e}$ are electron temperature and the electron density, $A(Z)$ is the abundance of the element, $G_{j \rightarrow i}^{(z)}\left(T, N_{e}\right)$ is the contribution function, which consists of two main terms, the photon emissivity coefficient $\left(P E C_{\sigma, j \rightarrow i}^{(e x c, z)}\right)$ to describe the atomic population of excited levels, with $\sigma$ the index of ground and metastable states, and the ionisation fraction $\left(N_{\sigma}^{(z)} / N^{(Z)}\right)$ calculated in equilibrium when $d N^{(z)} / d t=$ 0 , with $N_{\sigma}^{(z)}$ the number density in the ionisation stage z and $N^{(Z)}$ the total number density in all ionisation stages of a given element:

$$
G_{j \rightarrow i}^{(z)}\left(T, N_{e}\right)=\sum_{\sigma} P E C_{\sigma, j \rightarrow i}^{(e x c, z)}\left(N_{\sigma}^{(z)} / N^{(Z)}\right)_{e q}
$$

Note that only the contribution due to excitation has been included in equation (2). The full approach is described by [19].

The distribution of the electron temperature and density is specified by the $\Phi(T)$, which is called differential emission measure and depends on the square of electron density times the volume increment. 
As seen in equation (1) both the electron temperature and electron density enter the emission line intensity calculation. Therefore, one of the most common methods to measure those two quantities is the use of the intensity ratio of two spectral lines.

In particular, when the two lines originate from the same ion, the ratio between the line intensities (e.g. $I_{l}$ and $I_{2}$ ) can be simplified as following:

$$
\frac{I_{1}}{I_{2}}=\frac{\int G_{1}\left(T, N_{e}\right) \Phi(T) d T}{\int G_{2}\left(T, N_{e}\right) \Phi(T) d T}=\frac{P E C_{1}\left(T^{\text {peak }}, N_{e}\right)}{P E C_{2}\left(T^{\text {peak }}, N_{e}\right)}
$$

where $T^{\text {peak }}$ is temperature where the contribution function for the specific line peaks.

The comparison between the theoretical and observed line ratio provides a measurement of the electron temperature or electron density of the emitting source.

\section{Transient modelling}

For spectroscopic studies of dynamic finite density plasmas, it is crucial to focus on the lifetimes of the various states of atoms, ions and electrons. The order of these timescales, together with the plasma development times and their values relative to observation times, determines the modelling approach. The key lifetimes consists of two groups. The first group includes purely the atomic parameters (ground, metastable and excited state radiative decays and auto-ionising decay). The second group depends on plasma conditions (free particle thermalization, charge-state change, i.e. ionisation and recombination, and redistribution of population amongst excited states).

From a dynamic point of view, these two groups need to be compared with each other and with the plasma timescales, representing relaxation times of transient phenomena, plasma ion diffusion across temperature and density gradients and observations times.

In equilibrium, it is assumed that the ion population responds instantaneously to changes in the plasma temperature and density and the ionisation balance is calculated in ionisation equilibrium $\left(d N^{(z)} / d t=\right.$ $0)$.

Non-equilibrium occurs when the plasma electron temperature and density change on timescales shorter than the ionisation/recombination timescale $\tau_{i o n / r e c}[15]$, which is the time to reach steady state equilibrium:

$$
\tau_{\text {ion } / r e c}=\tau^{t h}=\sum_{Z} 1 /(S+\alpha) N_{e}
$$

with $S$ and $\alpha$ the ionisation and recombination coefficients. They give the contribution to the growth rates for the ground state population, due to the effective ionisation, which includes direct and excitation/autoionisation contributions, and the effective recombination, which includes radiative, dielectronic and threebody contributions. The values of these coefficients are currently within ADAS database and available through OPEN-ADAS [13].

In non-equilibrium, the local ionisation balance is no longer determined exclusively by the local temperature and density conditions, but depends on the past history of the temperature, density and ionisation state of the plasma. Therefore, the assumption of ionisation equilibrium in calculating ionisation balance is not appropriate and time-dependent ion populations must be derived from the following equation:

$$
\frac{d N^{(z)}}{d t}=N_{e}\left[S^{(z-1)} N^{(z-1)}+\left(S^{(z)}+\alpha^{(z)}\right) N^{(z)}+\alpha^{(z+1)} N^{(z+1)}\right]
$$

The transient contribution function then becomes: 


$$
G_{j \rightarrow i}^{(z)}\left(T_{e}, N_{e}, t\right)=P E C_{j \rightarrow i}^{(e x c, z)} \frac{N^{(z)}(t)}{N^{(Z)}}+P E C_{j \rightarrow i}^{(r e c, z)} \frac{N^{(z+1)}(t)}{N^{(Z)}}
$$

assuming that the PECs are not time-dependent as, in the examined plasma conditions, the excitation processes can be considered as instantaneous [e.g. 7, 8].

This is the case of flux tubes in the solar atmosphere, called loops, which contain condensation materials [14] or if there is an extremely rapid change in temperature [3]. Furthermore, it specifies the dynamic plasma behaviour in tokamak divertors, transport barriers and transients events in fusion devices.

In the following sections, this transient modelling is applied to analyse the electron temperature and density behaviour of a solar flare observed by the Ultraviolet Spectrometer and Polarimeter (UVSP) onboard of the Solar Maximum Mission (SMM) (Sect. 3.1.1) and in the context of the MAST Super-X divertor (Sect. 3.2.1), being fusion and astrophysics mutually supportive in such development. Furthermore, the same transient modelling together with hydrodynamic simulations is adopted as tool to diagnose the transient status of the plasma from observations taken by IRIS (Sect. 3.1.2).

\subsection{Solar atmosphere applications}

The solar atmosphere contains highly dynamic features such as flares, spicules, jets. However, these events require not only high spatial and spectral resolution but also high cadence to be observed. In particular, high cadence is generally not available for most of the UV spectrographs, due to constraints in instrument design. The only highest cadence (down to $1 \mathrm{~s}$ ) UV spectrometer before the launch of IRIS [5] in 2013 was, in fact, the UVSP [22] onboard of the Solar Maximum Mission in the early 1980s.

The high cadence spectral data acquired by those instruments have the potential to provide diagnostic information on the plasma status, but they require transient ionisation considerations.

Two observation sets have been used in the following sections:

1. Flare observed in the O V $1371 \AA \AA$ line using UVSP on $2^{\text {nd }}$ November 1980 [18].

2. Flare observed in the Si IV $1349 \AA$ and O IV $1401 \AA$, using IRIS time series on $2^{\text {nd }}$ April 2014.

\subsubsection{Deriving Te and Ne from a transient modelling}

Figure 1 (a) shows the O V $1371 \AA$ intensity during the impulsive phase of a flare as a function of time, with individual pulses, called bursts, labelled 1 to 10 . Each burst is treated as an independent entity, which is assumed to originate as a consequence of plasma heating at the foot of a loop in a multiple loop structure. Each of them has been fitted using the 'smooth-burst model' analytical expression [6]:

$$
f(t)=N^{o b} B^{R} e^{-\frac{\left(t-t_{s}\right)}{\tau^{o b}}}
$$

where $B=\left(t-t_{S}\right) e^{1} R^{-1} / \tau^{o b}, \tau^{o b}$ is the decay time, $t_{S}$ is the start time of the burst, $N^{o b}$ is the peak line enhancement value of the burst and $R$ controls the width of the burst.

The ionisation/recombination timescales (Eq. 4), $\tau^{\text {th }}$, for $\mathrm{O} \mathrm{V}$ have been calculated for different pairs of electron temperature and density. A theoretical peak line enhancement factor value $N^{\text {th }}$ has been derived using the transient modelling. It represents the theoretical line enhancement at the peak of the burst with respect to the equilibrium value. Finally the sets of $\left(T_{e}, N_{e}\right)$ pairs for each burst have been derived comparing the theoretical and observed peak line enhancements and timescales, as shown is Figure 1 (b). The final electron temperature and densities are plotted in Figure 1 (c) and (d). 

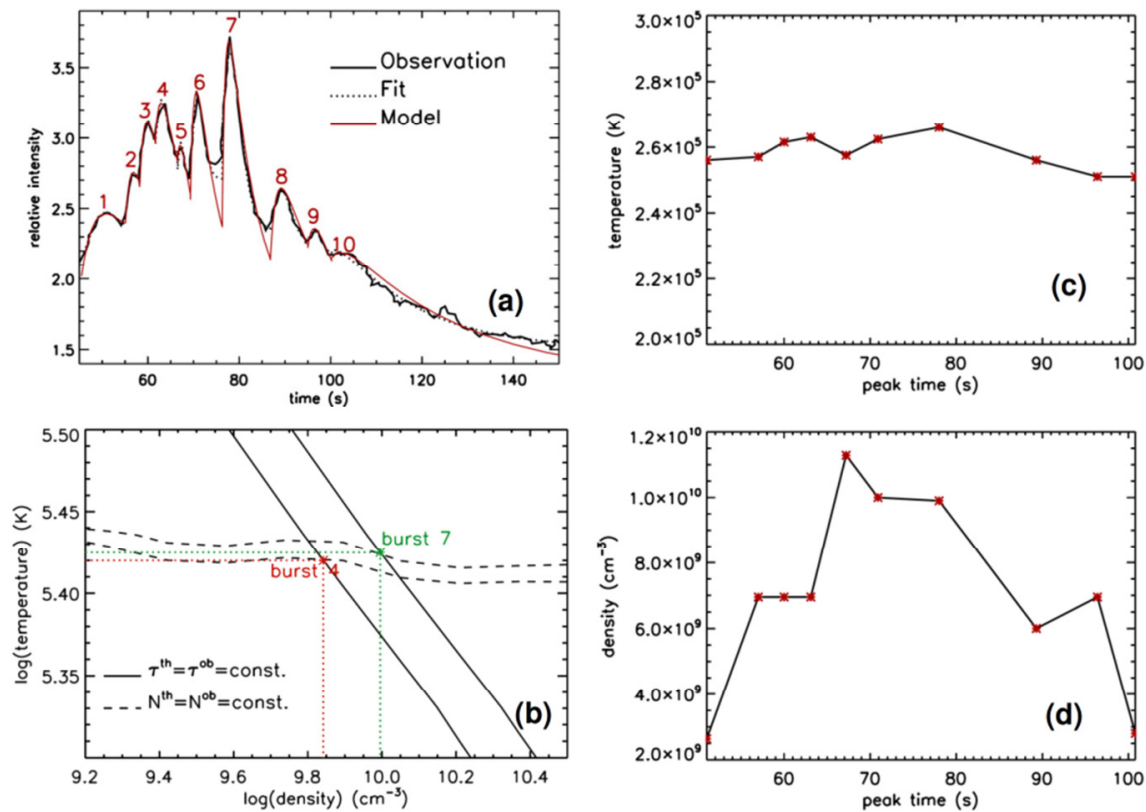

Figure 1- Method to derive electron temperature and electron density (c, d) from UV observation of a flare on $2^{\text {nd }}$ November 1980 using UVSP (a) as fully described in [7]. The relative intensity in Fig. 1 (a) is the intensity calculated using the transient modelling with respect to the intensity derived in equilibrium conditions. The method allows reconstructing each burst using the transient modelling and deriving from the comparison with the observational fit the values of electron temperature and density. In particular Fig. 1 (b) shows how burst 4 and 7 are obtained. The solid lines are the lines with $\tau$ constant, corresponding to the decay times of the fourth and seventh bursts, while the dashed lines have $N$ constant, corresponding to the peak values of the two considered bursts. The intersection points give the derived $T_{e}$ and $N_{e}$.

\subsubsection{Diagnosing transient ionisation from observations}

The diagnostic potential of high cadence observations made by IRIS has been discussed by [8]. The response of Si IV $1349 \AA$ and O IV $1401 \AA$ to non-equilibrium ionisation has been investigated using numerical simulation of coronal loop hydrodynamics [21], which reproduce a flaring small-scale event, and comparing them with IRIS observations of $2^{\text {nd }}$ April 2014.

Figure 2 (a) shows the ratio between the transient, $a_{3}=N^{(z)} / N^{(Z)}(t)$, and equilibrium, $a_{e q}=\left(N^{(z)} / N^{(Z)}\right)_{e q}$, ionisation balance calculations as a function of the loop length $s$ when the heating is turned on for Si IV and O IV. Si IV ionisation balance is enhanced at the loop footpoints by a factor $\sim 10$ compared to equilibrium, while O IV by a factor $\sim 3$. This shows that Si IV depends strongly on non-equilibrium ionisation and $\mathrm{O} I V$, instead, is less affected.

This different response of the two lines to transient ionisation may be directly compared with IRIS observations investigating the changes of their intensities during the flaring event with respect to a quiet region. Assuming that in the quiet Sun region both ions are in ionisation equilibrium, whereas the rapid variations of plasma conditions due to the flare lead to departure from ionisation equilibrium, the relation between the observed intensities and the theoretical modelling is given by:

$$
\frac{I_{A R}}{I_{Q S}}=\frac{\int G\left(T, N_{e}, t\right) d T}{\int G\left(T, N_{e}\right) d T}=\frac{\operatorname{PEC}\left(T^{\text {peak }}, N_{e}\right) a_{z=3}\left(T^{\text {peak }}, N_{e}, t\right)}{\operatorname{PEC}\left(T^{\text {peak }}, N_{e}\right) a_{e q}\left(T^{\text {peak }}, N_{e}\right)}=\frac{\alpha_{3}}{\alpha_{e q}}
$$

where $I_{A R}$ and $I_{Q S}$ are the line intensities in the flaring and quiet regions respectively. Figure 2 (b) displays the ratios between the intensities of Si IV and O IV of the flaring and quiet regions as a function of time, 
showing an enhancement of a factor 7 up to 23 for $\mathrm{Si} \mathrm{IV}$, and an enhancement of a factor 4 up to 10 for $\mathrm{O}$ IV intensities, which can be compared with the enhancement due to non-equilibrium of the hydrodynamic simulations. This might also affect the measurement of electron density of a transient feature when using $\mathrm{Si}$ IV and O IV ratio, leading to an over-estimate of up to a factor five.
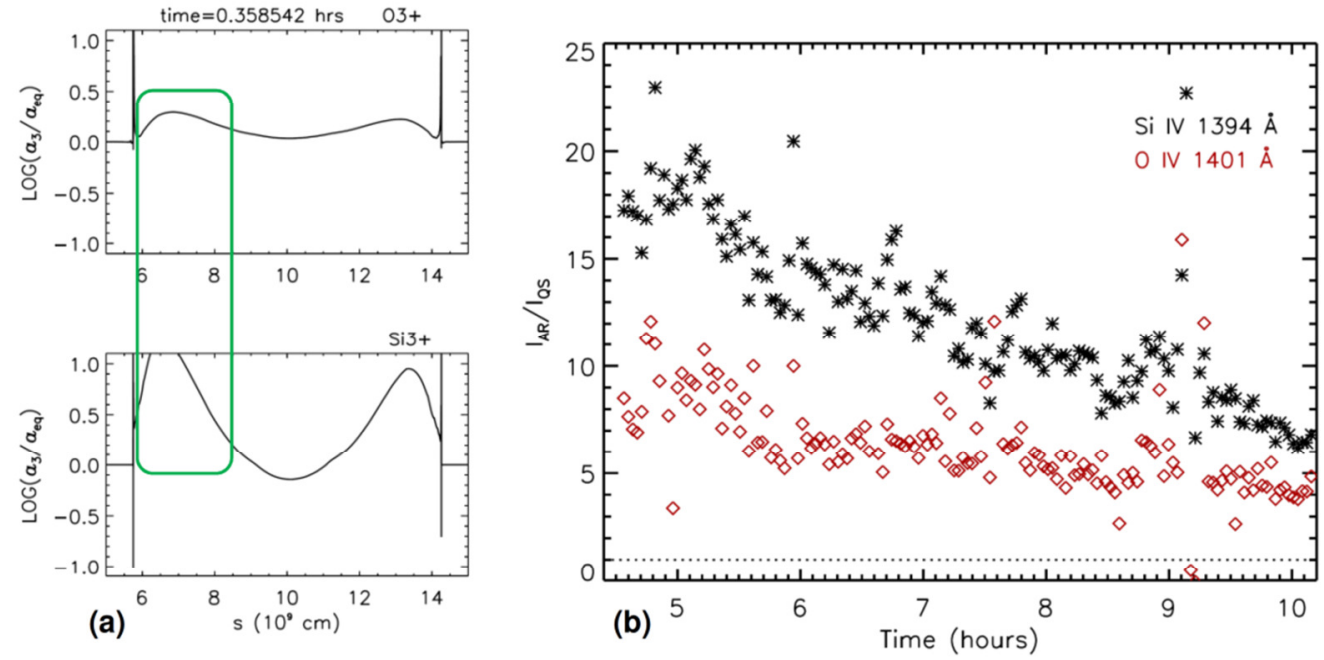

Figure 2 - (a) Non-equilibrium/equilibrium population ratios as a function of the loop length for the $\mathrm{O}^{3+}$ and $\mathrm{Si}^{3+}$ ions, referred in the text using the spectroscopic notation, O IV and Si IV respectively. (b) Intensity ratio of the flaring $\left(I_{A R}\right)$ and quiet $\left(I_{Q S}\right)$ regions for Si IV and O IV as observed by IRIS on $2^{\text {nd }}$ April 2014.

\subsection{Controlled fusion applications}

In this section attention is given to the transient modelling applied to tokamaks. In the layer of plasma surrounding the core plasma of such devices, called the Scrape-Off-Layer (SOL), filaments are often born as a result of the nonlinear saturation of underlying edge turbulence or magnetohydrodynamic (MHD) instabilities. These intermittent fluctuations provide a mechanism for the convective radial transport of particles, heat, momentum, and parallel current in the SOL. Furthermore, the timescales of these fluctuations are typically of the same order as magnitude as ionisation and recombination timescales. Therefore, a transient modelling is crucial in the investigation of the plasma properties.

In particular, Section 3.2.1 focusses on simulation of helium line emissivities in the visible wavelength range in a tokamak divertor. The potential diagnostic of helium line ratios are discussed, analysing the effect of transient ionisation on the plasma electron temperature and density measurements.

\subsubsection{Modelling transient ionisation in a tokamak divertor}

It is now well recognised that the lifetime of current divertor targets will be severely limited in future tokamaks, such as ITER. Moreover, the power deposition from typical operating modes in ITER will exceed the steady state power handling capability of the divertor target. Previous studies have shown that the detached divertor regime reduces the plasma pressure and electron temperature at the divertor target which allows for higher radiative power fractions in the SOL.

The divertor design on MAST-Upgrade $[9,17]$ provides the first opportunity to investigate the effect of a Super-X configuration, in which access to the detached regime is largely aided by the increased connection length to the divertor [12].

For deriving electron temperature and densities it has been noted that during the onset of detachment, line ratios from the Balmer series show an increase, being clear indicator of very low electron temperature $(\leq$ $1 \mathrm{eV}$ ). At higher temperatures (generally between 10-100 eV), He I line ratios of the $7283 \AA / 7067 \AA$ 
(from triplet and singlet spin systems) and $6680 \AA / 7283 \AA$ (from singlet spin system) transitions can be used as a gauge of electron temperature and the electron density respectively.
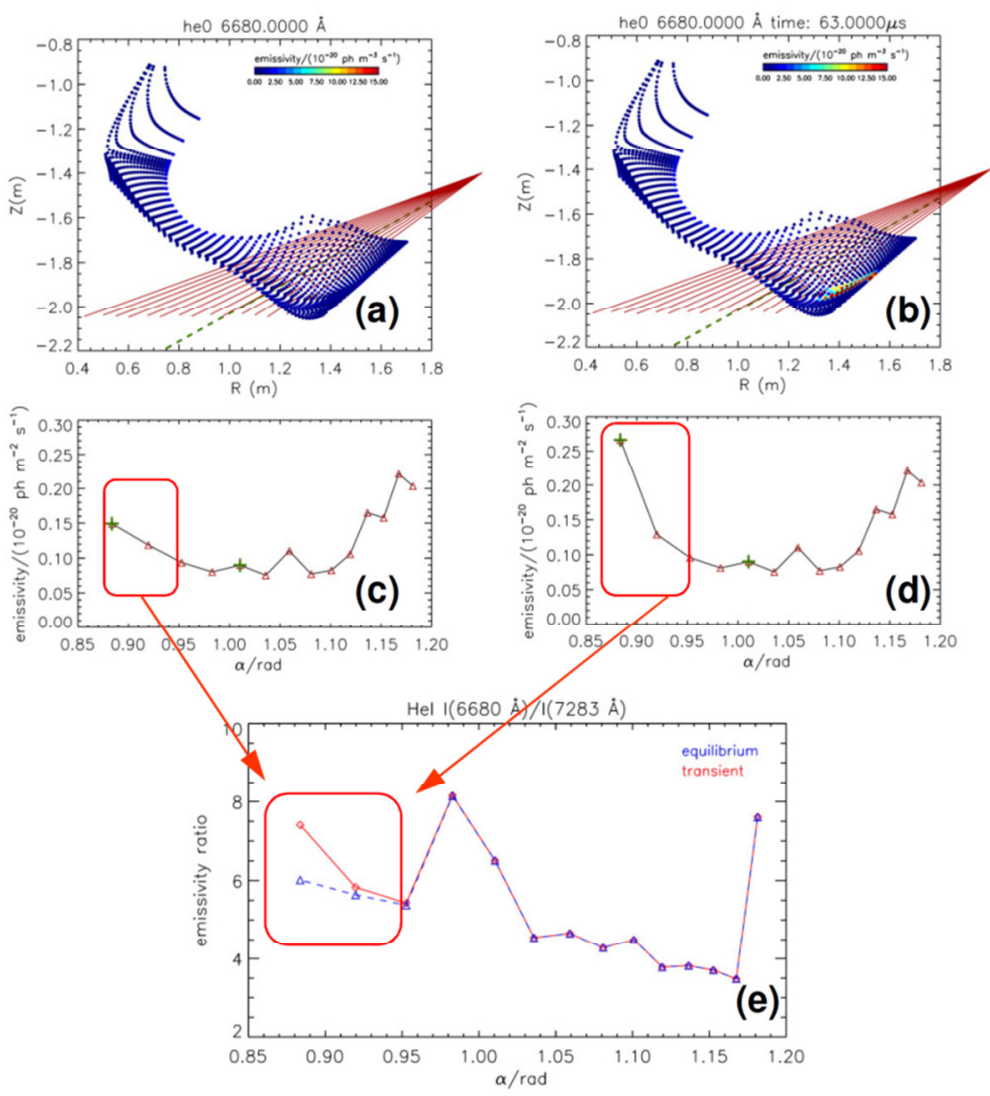

Figure 3 - Emissivity of He I line at $6680 \AA$ using B2-SOLPS simulations of Mast-Upgrade Super-X divertor in the case of equilibrium ionisation balance (a) and transient ionisation balance (b) calculations, with line of sight emissivity respectively (c, d). Density sensitive line ratio of $\mathrm{He}$ I between the two singlet lines at $6680 \AA$ and $7283 \AA$ derived using the equilibrium and transient modelling (e).

Figure 3 shows B2-SOLPS (SOL Plasma Simulations) of He I 6680 Å line emissivity when equilibrium ionisation is considered (Fig. 3 (a)) and when the transient ionisation modelling is applied (Fig. 3 (b)).

The emissivity has been integrated along each line of sight displayed in the plots and illustrated in Figure 3 (c) and (d).

Figure 3 (e) shows the effects of transient ionisation on the line ratio He I $6680 \AA$ A/7283 , suggesting that it might affect density measurement of the plasma in the vicinity of the Super-X divertor plate.

\section{Discussion and conclusions}

The spectroscopic diagnostic applications described in this work highlight that most of the plasma information is in the spectrum, ranging from plasma properties such as electron and ion temperature and density and the dynamic status to the atomic processes which give rise to the observed emission. However, extracting it requires careful modelling. A particularly crucial issue is that the response of the emission to a finite density environment and transients is similar. Therefore it is essential to model accurately both aspects for a consistent analysis of the observed plasma source, whether it originates from the space or in laboratory. 
Furthermore, new space-borne instrumentation, in particular SPICE on Solar Orbiter, as well as new tokamak devices, such as ITER and DEMO, entail continuous developments and update of atomic data and modelling to avoid interpretation error when analysing observations or measurements and to fulfil the mission requirements.

In this context, it is essential the synergy between astrophysics and laboratory plasmas, in order to implement and validate the common diagnostic techniques, such as the transient modelling, which can be developed with the mutual support of these two environments.

\section{Acknowledgments}

This work acknowledges the in house research support provided by the Science and Technology Facilities Council in UK and the participation in the International Team 276 funded by the International Space Science Institute (ISSI) in Bern, Switzerland. Armagh Observatory is funded by the N. Ireland Department of Communities.

\section{References}

[1] N. R. Badnell, et al., 2016, J. Phys. B: At. Mol. Opt. Phys., 49, 094001

[2] D. R. Bates, et al., 1962, Proc. Roy. Soc. A, 267, 297.

[3] S. J. Bradshaw \& H. E. Mason, 2003, Astron. Astrophys., 401, 699.

[4] A. Burgess \& H. P. Summers, 1969, Astrophys. J., 157, 1007.

[5] B. De Pontieu, et al., 2014, Solar Phys., 289, 2733.

[6] J. G. Doyle, et al., 1991, Mon. Not. Roy. Astron. Soc., 248, 503.

[7] J. G. Doyle, et al., 2012, Solar Phys., 280, 111

[8] J. G. Doyle, et al., 2013, Astron. Astrophys., 557, L9.

[9] G. Fishpool, et al., 2013, J. Nucl. Mater., 438, S356.

10] A. S. Giunta, et al., 2012, Astron. Astrophys., 538, A88.

[11] A. S. Giunta, et al., 2015, Astrophys. J.,803, 66.

[12] E. Havlíčková, et al., 2015, Plasma Phys. Control. Fusion, 57, 115001.

[13] http://open-adas.ac.uk

[14] A. F. Lanza, et al., 2001, Astrophys. J., 547, 1116.

[15] R. W. P. McWhirter, et al., 1965, in Plasma diagnostic techniques, eds. Huddlestone \& Leonard (New York, London: Academic Press)

[16] R. O. Milligan, et al., 2012, Astrophys. J. Lett., 755, L16.

[17] W. Morris, et al., 2014, IEEE Trans. Plasma Sci., 42, 402.

[18] A. I. Poland, et al., 1984, Astrophys. J., 280, 457.

[19] H. P. Summers, et al., 2006, Plasma Phys. Control. Fusion, 48, 263.

[20] H. P. Summers \& M. H. Hooper, 1983, Plasma Phys., 25, 1311.

[21] R. Susino, et al., 2010, Astrophys. J., 709, 499

[22] B. E. Woodgate, et al., 1980, Solar Phys., 65, 73. 